\title{
High-resolution diode-laser spectroscopy on a fast beam of metastable atoms for detecting very rare krypton isotopes
}

\author{
A.I. Ludin ${ }^{1,2}$, B.E. Lehmann ${ }^{1}$ \\ ${ }^{1}$ Physics Institute, University of Bern, Sidlerstrasse 5, CH-3012 Bern, Switzerland \\ (Fax: + 41-31/631-4405, E-mail: BLEHMANN@phil.unibe.ch) \\ ${ }^{2}$ Lamont-Doherty Earth Observatory of Columbia University, Route 9W, Palisades, NY 10964, USA
}

Received: 25 August 1994/Accepted: 15 February 1995

\begin{abstract}
A fast krypton ion beam with an energy of $10 \mathrm{keV}$ is transferred through a mass filter and neutralized in a $\mathrm{Rb}$ - (or Cs-) vapour charge exchange cell. The emerging beam of metastable $\mathrm{Kr}$ atoms of a selected $\mathrm{Kr}$ isotope is collinearly irradiated with a tunable cw GaAlAs diode laser at $811 \mathrm{~nm}$. The spectrum of the $1 s_{5}-2 p_{9}$ transition covers $\sim 10 \mathrm{GHz}$ and consists of one line each for the five stable isotopes of $\mathrm{Kr}$ with even mass number and 15 hyperfine structure lines of ${ }^{83} \mathrm{Kr}$. The individual lines are recorded by detecting the fluorescence signal perpendicular to the beams. Photons are guided to the PM tube by diffuse reflection from a high reflectance thermoplastics light collector. Up to 20 photons per atom are emitted by cycling between the two states during the flight time of $1.2 \mu$ s through the detector. The observed absorption linewidth of $100 \mathrm{MHz}$ is a combination of laser linewidth and energy uncertainty in the fast atomic beam. In order to achieve maximum isotope selectivity the beam energy has to be adjusted in such a way that the Doppler shifted lines of all isotopes form an optimum pattern for detecting the very rare isotopes ${ }^{81} \mathrm{Kr}$ and ${ }^{85} \mathrm{Kr}$ in natural environmental samples.
\end{abstract}

PACS: $07.65 ; 07.75 ; 32.00$

The two rare krypton radionuclides ${ }^{81} \mathrm{Kr}$ (half-life $210000 \mathrm{yr}$ ) and ${ }^{85} \mathrm{Kr}$ (half-life $10.76 \mathrm{yr}$ ) occur in $\mathrm{Kr}$ samples extracted from air, water or ice with relative isotopic abundances in the range of $10^{-11}$ to $10^{-14}[1-3]$. While ${ }^{85} \mathrm{Kr}$ can be detected in such samples by low level decay counting techniques $[4,5]$ on $\mathrm{Kr}$ samples from several hundred litres of water, no such technique exists for ${ }^{81} \mathrm{Kr}$. Age dating of very old groundwater and polar ice samples, e.g., is not possible with conventional radioactivity counters.

Accelerator Mass Spectroscopy (AMS) which is very successfully used for other rare radionuclides of importance in geochemistry $\left({ }^{10} \mathrm{Be},{ }^{14} \mathrm{C},{ }^{26} \mathrm{Al},{ }^{36} \mathrm{Cl},{ }^{129} \mathrm{I}\right)[6]$ is not feasible for noble gas atoms because tandem accelerators operate with negative ions. A recent study with positive ions at the ATLAS linear accelerator facility at Argonne National Laboratory has investigated the possibilities of AMS for detecting ${ }^{39} \mathrm{Ar}$ and ${ }^{81} \mathrm{Kr}$ [7]. For ${ }^{81} \mathrm{Kr}$ the problem of isobaric interference from ${ }^{81} \mathrm{Br}$ needs further experimental work.

An analytical technique based on Resonance Ionization Spectroscopy (RIS) has been developed in recent years for detecting noble gas atoms with isotopic selectivity $[8,9]$. Successful measurements of ${ }^{81} \mathrm{Kr}$ extracted from $\sim 501$ of old groundwater were reported $[10,11]$. The technique makes use of element-selective laser excitation and ionization of the $\mathrm{Kr}$ gas in the source region of a time of flight mass spectrometer. Very impressive detection limits below 100 atoms of ${ }^{81} \mathrm{Kr}$ in less than $5 \mathrm{~min}$ were achieved [12]. A disadvantage of the new technique, however, is the need for several isotope enrichment steps prior to the final laser atom counting due to the fact that the detection lasers (at 116.5, 558.1 and $1064.0 \mathrm{~nm}$ ) are pulsed and therefore have bandwidths which are larger than isotope effects in the $\mathrm{Kr}$ spectrum.

In the present work, we report experimental results with collinear beam spectroscopy using $\mathrm{cw}$ infrared diode lasers irradiating a beam of fast metastable $\mathrm{Kr}$ atoms. Such an approach has been proposed by several groups [13-18]. In particular, the rare isotope ${ }^{3} \mathrm{He}[19]$ and ${ }^{90} \mathrm{Sr}$ [20] have both been detected by similar concepts at concentrations of $10^{-9}$ to $10^{-11}$ in natural environmental samples.

The key factor in this technique is the optical isotope shift caused by the Doppler effect between different $\mathrm{Kr}$ isotopes moving at the same energy (and therefore with different velocities).

\section{Experimental concept}

The overall concept is illustrated in Fig. 1. A plasma discharge ion source generates a beam of $\mathrm{Kr}$ ions, which are accelerated to $10 \mathrm{keV}$ and focused to pass a Wien filter (crossed $E$ - and $B$-fields). The mass selected ion beam with 


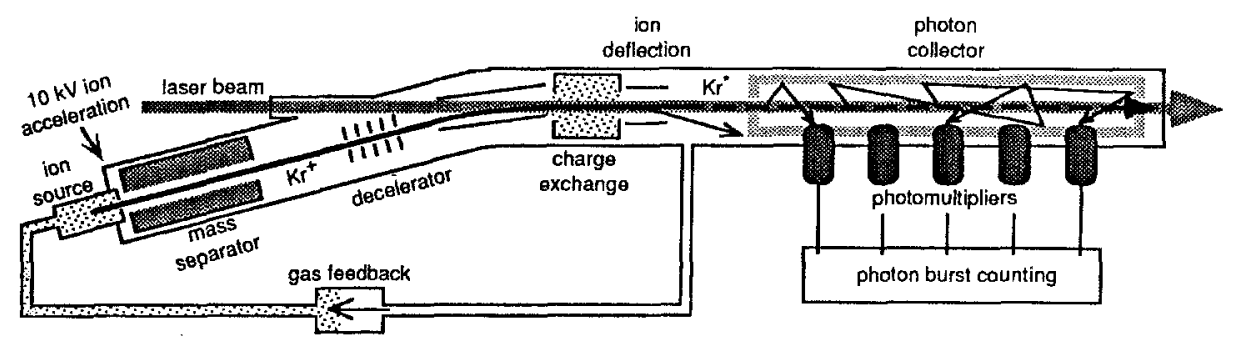

Fig. 1. Overview of the experimental concept. Data reported in this contribution were realized with a simpler test version (see text) a diameter of $\sim 1 \mathrm{~mm}$ is decelerated, deflected by a few degrees and enters an alkali vapour charge exchange cell for neutralization. A large fraction of the atoms is thereby transferred into a metastable stage. The remaining ions are deflected and the fast neutral atoms enter the detector where they are irradiated with tunable infrared laser light at $811 \mathrm{~nm}$. An important aspect of our arrangement is the closed vacuum system containing a small turbo pump to recycle the gas between the ion source and the rest of the system. This arrangement allows to work with a very small $\mathrm{Kr}$ sample of typically $3 \times 10^{-3} \mathrm{~cm}^{3} \mathrm{STP}$ (representing $\sim 301$ of groundwater) for several hours [21]. The steady-state pressures are $2 \times 10^{-2}$ mbar in the plasma discharge ion source and $2 \times 10^{-5}$ mbar in the rest of the detector system. Getters remove reactive gases.

The experimental results reported in this work were achieved with an earlier version of the detector where the ion decelerator and the small angle beam deflection were not yet realized. Therefore spectroscopic studies with counterpropagating beams at $10 \mathrm{keV}$ ion beam energy only have been possible so far. Furthermore, only one single photomultiplier and a short detector of $18 \mathrm{~cm}$ were in use for these first tests.

\section{Infrared spectroscopy on metastable $\mathrm{Kr}$ at $811.5 \mathrm{~nm}$}

A large fraction of the neutralized $\mathrm{Kr}$ atoms remains in the $1 s_{5}$ state due to the quasi-resonant charge exchange with alkali atoms [22]. The transition to the $2 p_{9}$ state is highly allowed and can easily be saturated with less than $100 \mu \mathrm{W}$ narrow band laser light over a beam diameter of $\sim 1 \mathrm{~mm}$.

At $10 \mathrm{keV}$ ion beam energy a ${ }^{84} \mathrm{Kr}$ atom moves at a velocity of $15 \mathrm{~cm} / \mu \mathrm{s}$. The Doppler isotope shift is $186.8 \mathrm{GHz}$ under these conditions. With counterpropagating beams the laser wavelength has to be shifted by about $0.41 \mathrm{~nm}$ to the red to compensate for the blue shift between atoms and photons. The exact line positions can be calculated from the isotope shifts and hyperfine constants that have been known for some time for the stable $\mathrm{Kr}$ isotopes [23-25] but have been measured for this transition only very recently for ${ }^{81} \mathrm{Kr}$ and ${ }^{85} \mathrm{Kr}$ [26]. For ${ }^{81} \mathrm{Kr}$ a sample of ${ }^{80} \mathrm{Kr}$ was enriched in a reactor by neutron activation; for ${ }^{85} \mathrm{Kr}$ a high activity gas was separated from nuclear fission products. With these new data it is now possible to exactly calculate the full $\mathrm{Kr}$ spectrum from all natural stable and radioactive $\mathrm{Kr}$ isotopes.

Table 1 summarizes the calculated isotope shifts relative to ${ }^{84} \mathrm{Kr}$ for all lines of the even nuclides and the
Table 1. Calculated isotope shifts for all $\mathrm{Kr}$ isotopes relative to ${ }^{84} \mathrm{Kr}$

\begin{tabular}{|c|c|c|c|}
\hline Line no. & Isotope & $\begin{array}{l}\text { Hyperfine } \\
\text { transition }\end{array}$ & $\begin{array}{l}\text { Frequency } \\
\text { shift }[\mathrm{MHz}]\end{array}$ \\
\hline 1 & 83 & $7 / 2-5 / 2$ & -1408 \\
\hline 2 & 85 & $7 / 2-5 / 2$ & -1270 \\
\hline 3 & 83 & $5 / 2-5 / 2$ & -1147 \\
\hline 4 & 83 & $9 / 2-7 / 2$ & -1132 \\
\hline 5 & 81 & $5 / 2-3 / 2$ & -1074 \\
\hline 6 & 85 & $5 / 2-5 / 2$ & -1067 \\
\hline 7 & 85 & $9 / 2-7 / 2$ & -1060 \\
\hline 8 & 81 & $1 / 2-3 / 2$ & -1032 \\
\hline 9 & 81 & $3 / 2-3 / 2$ & -1029 \\
\hline 10 & 81 & $7 / 2-5 / 2$ & -1008 \\
\hline 11 & 83 & $3 / 2-5 / 2$ & -979 \\
\hline 12 & 85 & $3 / 2-5 / 2$ & -953 \\
\hline 13 & 81 & $5 / 2-5 / 2$ & -837 \\
\hline 14 & 81 & $9 / 2-7 / 2$ & -800 \\
\hline 15 & 81 & $3 / 2-5 / 2$ & -792 \\
\hline 16 & 83 & $7 / 2-7 / 2$ & -753 \\
\hline 17 & 85 & $7 / 2-7 / 2$ & -724 \\
\hline 18 & 83 & $11 / 2-9 / 2$ & -706 \\
\hline 19 & 85 & $11 / 2-9 / 2$ & -688 \\
\hline 20 & 85 & $5 / 2-7 / 2$ & -521 \\
\hline 21 & 83 & $5 / 2-7 / 2$ & -492 \\
\hline 22 & 81 & $7 / 2-7 / 2$ & -397 \\
\hline 23 & 81 & $11 / 2-9 / 2$ & -309 \\
\hline 24 & 81 & $5 / 2-7 / 2$ & -227 \\
\hline 25 & 78 & & -217 \\
\hline 26 & 83 & $9 / 2-9 / 2$ & -176 \\
\hline 27 & 85 & $9 / 2-9 / 2$ & -164 \\
\hline 28 & 80 & & -138 \\
\hline 29 & 83 & $13 / 2-11 / 2$ & -87 \\
\hline 30 & 85 & $13 / 2-11 / 2$ & -77 \\
\hline 31 & 82 & & -64 \\
\hline 32 & 84 & & 0 \\
\hline 33 & 86 & & 66 \\
\hline 34 & 85 & $7 / 2-9 / 2$ & 171 \\
\hline 35 & 83 & $7 / 2-9 / 2$ & 203 \\
\hline 36 & 81 & $9 / 2-9 / 2$ & 463 \\
\hline 37 & 83 & $11 / 2-11 / 2$ & 635 \\
\hline 38 & 81 & $13 / 2-11 / 2$ & 656 \\
\hline 39 & 85 & $11 / 2-11 / 2$ & 703 \\
\hline 40 & 83 & $15 / 2-13 / 2$ & 783 \\
\hline 41 & 81 & $7 / 2-9 / 2$ & 866 \\
\hline 42 & 85 & $15 / 2-13 / 2$ & 870 \\
\hline 43 & 83 & $9 / 2-11 / 2$ & 1166 \\
\hline 44 & 85 & $9 / 2-11 / 2$ & 1226 \\
\hline 45 & 83 & $13 / 2-13 / 2$ & 1744 \\
\hline 46 & 81 & $11 / 2-11 / 2$ & 1967 \\
\hline 47 & 85 & $13 / 2-13 / 2$ & 1986 \\
\hline 48 & 83 & $11 / 2-13 / 2$ & 2466 \\
\hline 49 & 81 & $9 / 2-11 / 2$ & 2739 \\
\hline 50 & 85 & $11 / 2-13 / 2$ & 2766 \\
\hline
\end{tabular}


hyperfine manifolds of the odd nuclides ${ }^{81} \mathrm{Kr},{ }^{83} \mathrm{Kr}$ and ${ }^{85} \mathrm{Kr}$. A total of 50 transition lines results. For detecting the rare isotopes ${ }^{81} \mathrm{Kr}$ and ${ }^{85} \mathrm{Kr}$ only their two strongest transitions will most likely be of importance; they are shifted by $+656 \mathrm{MHz}(13 / 2-11 / 2)$ for ${ }^{81} \mathrm{Kr}$ and by $+870 \mathrm{MHz}(15 / 2-13 / 2)$ for ${ }^{85} \mathrm{Kr}$ relative to the ${ }^{84} \mathrm{Kr}$ line. It is important to note that both these transitions occur between a perfect two-level system. Excited atoms have no choice but to decay back to the lower state, and therefore atoms can be cycled between the two states by a cw laser beam. With a radiative lifetime of $30 \mathrm{~ns}$ [27, 28], an estimated cycling time of $60 \mathrm{~ns}$, each atom will re-emit just about one photon per $\mathrm{cm}$ of path (at $10 \mathrm{keV}$ beam energy). At a lower beam energy of e.g. $200 \mathrm{eV}$ and with a possible detector length of (e.g.) $100 \mathrm{~cm}$, up to 700 photons could be re-emitted by each atom while passing through such a long detector. This is an important requirement for a possible detection scheme based on photon burst discrimination [14].

\section{The $\mathrm{Kr}$ spectrum at $10 \mathrm{keV}$ with counterpropagating beams}

In Fig. 2 we present the measured $\mathrm{Kr}$ spectrum at $10 \mathrm{keV}$ for the even nuclides (top) and for the odd ${ }^{83} \mathrm{Kr}$ isotope (middle) together with the calculated relative Doppler shift of all lines as a function of the ion acceleration voltage (bottom). The top spectrum was recorded by setting the mass filter to mass 78 , followed by scanning the laser over the ${ }^{78} \mathrm{Kr}$ absorption line. The mass filter was then subsequently adjusted to the krypton isotope with the next higher mass before continuing the stepwise laser tuning for each absorption line. For the recording of the ${ }^{83} \mathrm{Kr}$ hyperfine structure spectrum (middle) the Wien filter was set to mass 83 . Small fractions of the neighbouring isotopes ${ }^{82} \mathrm{Kr}$ and ${ }^{84} \mathrm{Kr}$ still were allowed to pass the mass filter in order to get markers at the corresponding wavelengths. The calculated isotope shifts for different beam energies (bottom of the figure) are relative to the ${ }^{81} \mathrm{Kr}$ hyperfine manifold at zero energy. In such a picture the strongest ${ }^{81} \mathrm{Kr}$ line $(F=13 / 2-F=11 / 2)$ which is shift-

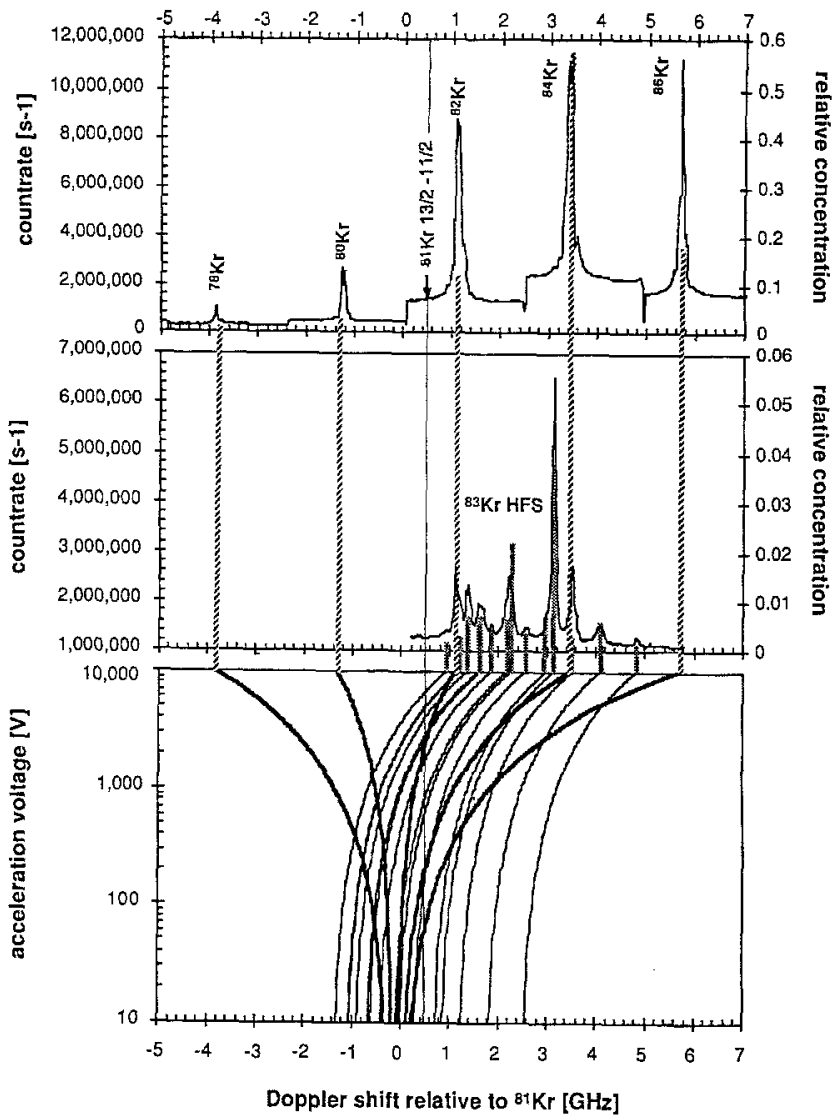

Fig. 2. Top: krypton spectrum at $10 \mathrm{keV}$ with counterpropagating beams, even nuclides; center: ${ }^{83} \mathrm{Kr}$ hyperfine manifold; bottom: calculated frequency shifts of all lines as a function of ion acceleration voltage relative to the ${ }^{81} \mathrm{Kr}$ lines

ed by $+656 \mathrm{MHz}$ relative to ${ }^{84} \mathrm{Kr}$ (Table 1) appears as a vertical line which crosses the curves of the various $\mathrm{Kr}$ isotopes illustrating the changing pattern of interference from more abundant isotopes at various beam energies.

In order to maximize optical isotope selectivity the ${ }^{81} \mathrm{Kr}$ line has to be moved away from other lines as far as possible by selecting the proper ion beam energy. In Fig. 3,

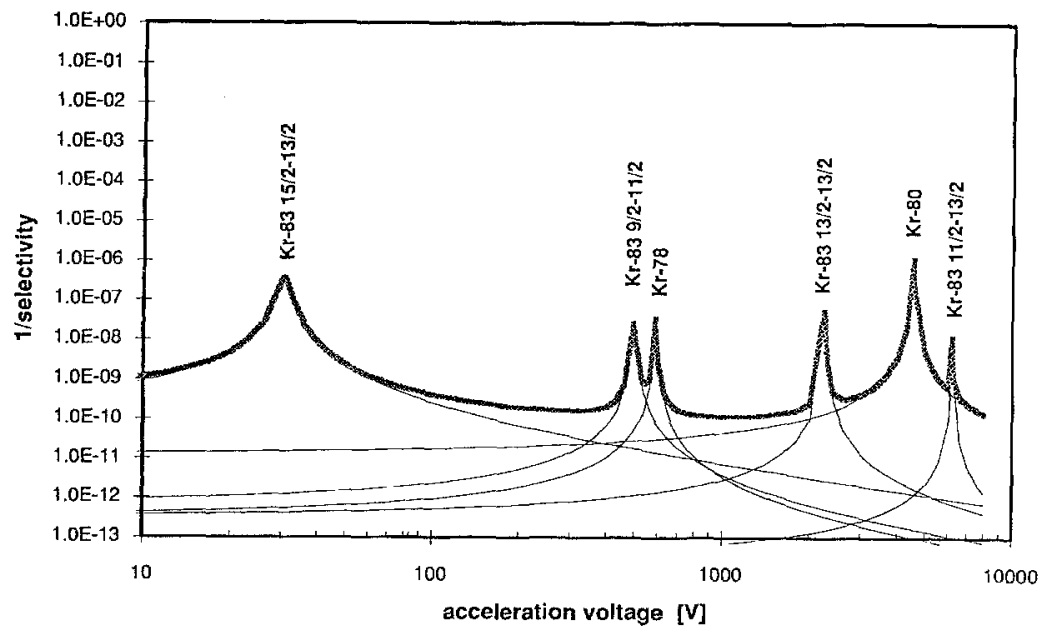

Fig. 3. Inverse selectivity for detecting the ${ }^{81} \mathrm{Kr}$ (13/2-11/2) absorption line calculated for copropagating beams and mass separation in a $15^{\prime \prime}$ Wien filter (see text) 
we have calculated the best regions of beam energy for the selective excitation of ${ }^{81} \mathrm{Kr}$. For copropagating beams, ranges between 100 and $400 \mathrm{eV}$, between 800 and $1200 \mathrm{eV}$ and above $9 \mathrm{keV}$ appear to be best suited for such work. For calculating the theoretical selectivities in Fig. 3 we have made the following assumptions:

(1) Wien filter abundance sensitivities relative to mass 81 are 20000 for ${ }^{80} \mathrm{Kr}$ and ${ }^{82} \mathrm{Kr}$ and 100000 for all other stable $\mathrm{Kr}$ isotopes. These factors were realized with a $15^{\prime \prime}$ filter by our colleagues in Oak Ridge [29]. The 6" Wien filter in our current experimental arrangement operates with factors that are 1 to 2 orders of magnitude lower.

(2) The line profiles are Lorentzian with a natural width (FWHM) of $5.3 \mathrm{MHz}$ corresponding to the excited-state lifetime of $30 \mathrm{~ns}$.

(3) All abundances of stable $\mathrm{Kr}$ isotopes are those of a natural $\mathrm{Kr}$ sample.

As can be seen in Fig. 3 the most interfering line for ${ }^{81} \mathrm{Kr}$ at $10 \mathrm{keV}$ as well as at $800 \mathrm{eV}$ is the ${ }^{80} \mathrm{Kr}$ line. Under optimal conditions the present concept should reach a combined isotope selectivity of mass filter and selective optical excitation of $10^{10}$.

\section{Sensitivity and selectivity}

To discuss the various factors which influence the sensitivity and selectivity of detection we refer to Fig. 4 . The ${ }^{78} \mathrm{Kr}$ line (with an isotopic abundance of $0.35 \%$ in atmospheric krypton) was recorded with a laser scanning of $10 \mathrm{MHz} /$ channel and a data accumulation time of $0.3 \mathrm{~s} /$ channel. The width of the line (FWHM) is approximately $100 \mathrm{MHz}$, the frequency scale being calibrated with a confocal Fabry-Perot interferometer with a free spectral range of $250 \mathrm{MHz}$. The "satellites" in the line profile must be caused by instabilities of the detection system. A $10 \mathrm{MHz}$ variation during the sampling time of $0.3 \mathrm{~s} \mathrm{can}$ be caused by:

(1) an energy fluctuation of $1 \mathrm{eV}$ of the ion beam (relative to $10000 \mathrm{eV}$ beam energy);

(2) a temperature instability of $0.33 \mathrm{mK}$ in the diode laser (at the operating temperature of $32^{\circ} \mathrm{C}$ and with a characteristic temperature tuning of $30 \mathrm{GHz} / \mathrm{K}$ );

(3) an instability of $3 \mu \mathrm{A}$ in the diode-laser current (at the operating current of $70 \mathrm{~mA}$ and with a current tuning rate of $3.3 \mathrm{GHz} / \mathrm{mA}$ ).

The spectrum was recorded with a neutral current at mass 78 of $0.12 \mathrm{nA}$ (or $7.5 \times 10^{8}$ atoms/s). The recorded net count rate of $2.58 \mathrm{MHz}$ at the peak therefore corresponds to a detection efficiency of 0.0034 counts per ${ }^{78} \mathrm{Kr}$ ion (or $21.5 \mathrm{MHz} / \mathrm{nA}$ ). This overall efficiency is a product of the following factors: $f_{1}$ : efficiency of charge exchange of ions into the metastable $1 s_{5}$ state; $f_{2}$ : efficiency of optical excitation, mainly determined by the geometrical overlap of laser beam and atomic beam including their respective shapes; $f_{3}$ : efficiency of light collection from the linear source of $18 \mathrm{~cm}$ length; $f_{4}$ : photomultiplier efficiency at $811.5 \mathrm{~nm} ; f_{5}$ : maximum number of

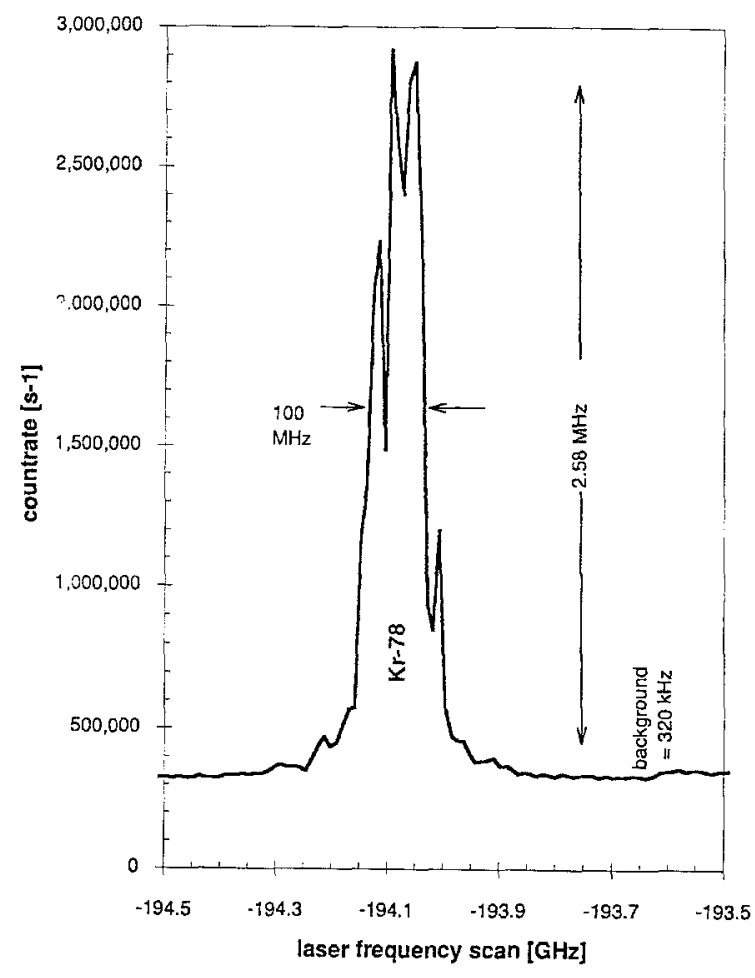

Fig. 4. ${ }^{78} \mathrm{Kr}$ absorption line, counterpropagating beams at $10 \mathrm{keV}$

photons re-emitted per atom during the flight time through the detector.

The individual contributions cannot easily be determined separately with the present set-up. The following values are our current best guesses:

$$
\begin{aligned}
f_{1} \times f_{2} \times f_{3} \times f_{4} \times f_{5} & =45 \% \times 30 \% \times 7 \% \times 1.8 \% \times 20 \% \\
& =0.0034 \text { photons/atom } .
\end{aligned}
$$

The following comments must be made: The measured neutralization efficiency can reach $80 \%$ or more but it is currently not known what fraction of the atoms is converted to the $1 s_{4}$ state (which would be lost for the detection) and what fraction may even be lost to the ground state, e.g., by collisions. The quoted overlap efficiency of $f_{3}=30 \%$ between atomic and laser beam certainly can be improved; however, in practice it is not easy to exactly overlap an infrared laser beam with a beam of neutral atoms while minimizing the stray light of the laser of the photomultiplier. A factor of $f_{2}=100 \%$ would represent a situation where every metastable atom re-emits the maximum of 20 photons on its way through the detector. The light collection efficiency was realized in a prototype detector using multiple diffuse reflections in a high reflectance thermoplastics (Spectralon, reflectance $=99.1 \%$ at $800 \mathrm{~nm}$ ) to guide photons from the inner walls to the photomultiplier which has an active area of $17 \mathrm{~cm}^{2}$ (RCA 8852 ) and which is currently positioned at a distance of $16 \mathrm{~cm}$ from the beam axis. Unfortunately the photocathode responsivity in this spectral region is at most $2 \%$. Although we have no direct proof of the cycling of atoms between the two hyperfine levels, we consider the measured detection efficiency presented above together with the best guess of values for the various factors as indirect 
evidence that cycling actually occurs. Without any cycling unrealistically higher values for other parameters would otherwise have to be postulated.

Other parameters which are important for a future use of such a technique for ${ }^{81} \mathrm{Kr}$ analysis can also be taken from Fig. 4. The background count rate of $320 \mathrm{kHz}$ has three components:

(1) photomultiplier dark count rate $=4 \mathrm{kHz}$ (cooled to approx. $5^{\circ} \mathrm{C}$ );

(2) count rate from photon emission caused by collisions between fast atoms and residual gas molecules in the system is $36 \mathrm{kHz}$ (equivalent to $\sim 500 \mathrm{kHz}$ per nA neutral current) and

(3) stray light $=280 \mathrm{kHz}$. This last component currently limits the detection sensitivity of our system and needs to be decreased in the next steps of system development. With the small angle ion beam deflection it will be possible to exactly guide the laser beam through the vacuum system and absorb the energy in a better defined target than in the present preliminary arrangement.

\section{Outlook}

It is our goal to increase the detection sensitivity to a level above 1 photon/atom in order to be able to apply the discrimination methods demonstrated for photon burst detection $[16,17,30,31]$. By slowing down the atoms to $200 \mathrm{eV}$ and increasing the detector length to $100 \mathrm{~cm}$ each atom will be exposed to photons 40 times longer than in the present experiment. A maximum of 800 photons can be re-emitted by each atom under these conditions. The second-generation light collector which is currently under construction therefore has to reach an overall photon collection efficiency of at least $50 \%$.

It is anticipated that in the final version of a detector for the rare noble gas nuclides ${ }^{81} \mathrm{Kr}$ and ${ }^{85} \mathrm{Kr}$ in environmental krypton samples the required isotope selectivity of $10^{12}$ or better will be distributed in about equal parts to (1) isotope separation in the ion mass filter, (2) optical isotope selectivity in the Doppler shifted spectra of fast metastable atoms and (3) photon burst discrimination against remaining more abundant stable atoms and stray light.

Acknowledgements. This project was supported by the Swiss National Science Foundation and the State of Bern. The authors thank B. Bushaw, B. Cannon, H. Loosli, K. Niemax, N. Thonnard, $\mathrm{K}$. Wendt and T. Whitaker for very helpful comments and suggestions and K. Grossenbacher, H.P. Moret and H. Riesen for their technical skills.

\section{References}

1. H.H. Loosli, H. Oeschger: Earth Planet. Sci. Lett. 7, 67 (1969)

2. V.V. Kuzminov, A.A. Pomansky: In Proc. 18th Int'l Cosmic Ray Conf., Bangalore, Vol. 2 (1983) p. 357

3. B.E. Lehmann, H.H. Loosli: Inst. Phys. Conf. Ser. 71, 219 (1984)

4. W.M. Smethie, G. Mathieu: Marine Chem. 18, 17 (1986)

5. K. Rozanski, T. Florkowski: IAEA Proc. 2, 949 (1979)

6. R.C. Finkel, M. Suter: Adv. Anal. Geochem. 1, 1 (1993)

7. W. Kutschera, M. Paul, I. Ahmad, T.A. Antaya, P.J. Billquist, B.G. Glagola, R. Harkewicz, M. Hellstrom, D.J. Morrissey, R.C. Pardo, K.E. Rehm, B.M. Sherrill, M. Steiner: In Proc. 6th Int'l Conf. on Accelerator Mass Spectrometry, Canberra (1993)

8. G.S. Hurst, M.G. Payne, S.D. Kramer, C.H. Chen, R.C. Phillips, S.L. Allman, G.D. Alton, J.W. Dabbs, R.D. Willis, B.E. Lehmann: Rep. Prog. Phys. 48, 1333 (1985)

9. B.E. Lehmann, H. Oeschger, H.H. Loosli, G.S. Hurst, S.L. Allman, C.H. Chen, S.D. Kramer, M.G. Payne, R.C. Phillips: J. Geophys. Res. 90, B13, 11547 (1985)

10. N. Thonnard, R.D. Willis, M.C. Wright, W.A. Davis, B.E. Lehmann: Nucl. Instrum. Methods B 29, 398 (1987)

11. B.E. Lehmann, H.H. Loosli, N. Thonnard, R.D. Willis: Appl. Geochem. 6, 419 (1991)

12. N. Thonnard, M.C. Wright, W.A. Davis, R.D. Willis: Inst. Phys. Conf. Ser. 128, 27 (1992)

13. B.D. Cannon, T. Whitaker: Appl. Phys. B 38, 57 (1985)

14. W.M. Fairbank Jr.: Nucl. Instrum. Methods B 29, 407 (1987)

15. S.A. Aseyev, Yu.A. Kudryavtsev, V.S. Letokhov, V.V. Petrunin: J. Phys. B 24, 2755 (1991)

16. W.M. Fairbank Jr., C.S. Hansen, R.D. La Belle, X.-J. Pan, E.P. Chamberlin, B.L. Fearey, R.E. Gritzo, R.A. Keller, C.M. Miller, H. Oona: SPIE Proc. 1435, 86 (1991)

17. E.C. Benck, H.A. Schuessler, F. Buchinger, K. Carter: Inst. Phys. Conf. Ser. 128, 329 (1992)

18. B.E. Lehmann, A. Ludin: Inst. Phys. Conf. Ser. 128, 41 (1992)

19. S.A. Aseyev, Yu.A. Kudryavtsev, V.S. Lethokov, V.V. Petrunin: Opt. Lett. 16, 514 (1991)

20. K. Zimmer, J. Stenner, H.-J. Kluge, J. Lantzsch, L. Monz, E.W. Otten, G. Passler, R. Schwalbach, M. Schwarz, H. Stevens, K. Wendt, G. Herrmann, S. Niess, N. Trautmann, K. Walter, B.A. Bushaw: Appl. Phys. B 59, 117 (1994)

21. B.E. Lehmann, D.F. Rauber, N. Thonnard, R.D. Willis: Nucl. Instrum. Methods B 28, 571 (1987)

22. G.E. Ice, R.E. Olson: Phys. Rev. A 11, 111 (1975)

23. D.A. Jackson: J. Opt. Soc. Am. 69, 503 (1979)

24. H. Gerhardt, F. Jeschonnek, W. Makat, E. Matthias, H. Rinneberg, F. Schneider, A. Timmermann, R. Wenz, P.J. West: Hyperfine Interact. 9, 175 (1981)

25. B.D. Cannon, G.R. Janik: Phys. Rev. A 42, 397 (1990)

26. B.D. Cannon: Phys. Rev. A 47, 1148 (1993)

27. M.V. Fonseca, J. Campos: Phys. Rev. A 17, 3 (1978)

28. R.S.F. Chang, H. Horiguchi, D.W. Setser: J. Chem. Phys. 73, 2 (1980)

29. W.A. Davis, N. Thonnard: Inst. Phys. Conf. Ser. 94, 223 (1988)

30. D.A. Lewis, J.F. Tonn, S.L. Kaufmann, G.W. Greenlees: Phys. Rev. A 19, 1580 (1979)

31. R.D. LaBelle, W.M. Fairbank Jr., R.A. Keller: Phys. Rev. A 40, 5430 (1989) 